\title{
Introduction to the AI and the Future of Work Minitrack
}

\author{
Triparna de Vreede, Xusen Cheng \\ University of South Florida, Renmin University of China \\ tdevreede@usf.edu, xusen.cheng@,ruc.edu.cn
}

Developments in Artificial Intelligence (AI) are setting the stage for a paradigm shift in the workplace. According to $\mathrm{PwC}, 72 \%$ of business executives say that $\mathrm{AI}$ will give them a competitive advantage in the future [1]. In fact, in many ways the future is already here. Several repetitive and simple tasks that were typically performed by humans are now being automated by AI bundled with other technologies, such as cloud systems, augmented reality, (social) robots, IoTs, and wearables. Consequently, the ratio of tasks executed by machines to humans is dramatically shifting. Human workers are rapidly being replaced with AI on many fronts. For instance, in 2018 an average of $71 \%$ of total task hours were performed by humans compared to $29 \%$ accomplished by machines. However, this average is expected to shift dramatically in the near future with $58 \%$ of tasks hours expected to be performed by humans and the remaining $42 \%$ to be handled exclusively by machines by 2022 [2].

In a world of such rapid technological changes, it is paramount to have an overview of the research being conducted in this discipline. Such an understanding of current developments of AI and associated technologies enables us to envision the future workplace and its effect on human employees. These insights also provide guidance to employees, teams, organizations, and governments in creating a sustainable and synergistic workplace. Further, engaging in this research allows us to proactively counter any negative effects that may arise from the changes in the work settings. Through this minitrack, we hope to create a platform where researchers working on $\mathrm{AI}$ in the context of future of work can come together and create collaborations that will allow them to capitalize on each other's work.

This minitrack focuses on the impact of AI on the various aspects of the workplace as it exists currently as well as how it may evolve in the future. We host papers that address the social, technical, behavioral, attitudinal, emotional, or managerial aspects of AI in the workplace. The unit of analysis can be individuals, teams, or organizations. The research focus can range from the impact of AI on work and its related aspects to the design considerations of AI in the workplace. In short, this minitrack highlights research that may influence the future of work and act as a springboard for new ideas and innovations in AI that will be disruptive to the workplace.

The first minitrack at HICSS-54 was highly successful and attracted several submissions despite the pandemic. The online session held was also very successful with approximately 38 attendees at its peak. This year's minitrack features the following five papers:

- Impact of Robotic Process Automation on Future Employment of Accounting Professionals, by Gladie Lui and Connie Shum.

- The AI Family: The Information Security Managers Best Frenemy?, by Kristel de Nobrega and AnneFrançoise Rutkowski.

- $\quad$ Task Delegability to AI: Evaluation of a Framework in a Knowledge Work Context, by Izabel Cvetkovic and Eva Bittner.

- Ethics Guidelines for Using AI-based Algorithms in Recruiting: Learnings from a Systematic Literature Review, by Lennart Hofeditz, Milad Mirbabaie, Audrey Luther, Riccarda Mauth, and Ina Rentemeister.

- Requirements for AI-based Teammates: A Qualitative Inquiry in the Context of Creative Workshops, by Edona Elshan, Dominik Siemon, Triparna de Vreede, Gert-Jan de Vreede, Sarah Oeste-Reiß, and Philipp Ebel.

\section{References}

[1] Bothun, D., Liebermann, M., and Rao, A. (2017) "Bot.Me: A revolutionary partnership. How AI is pushing man and machine closer together." Consumer Intelligence Series PwC. http://pwcartificialintelligence.com/

[2] World Economic Forum. (2018). "The future of jobs report 2018". Geneva: World Economic Forum.http://www3.weforum.org/docs/WEF Future of Jobs 2018.pdf 